Kardiologe 2020 · 14:106-112

https://doi.org/10.1007/s12181-020-00384-2

Online publiziert: 13. März 2020

(c) Deutsche Gesellschaft für Kardiologie Herz- und Kreislaufforschung e.V. Published by Springer Medizin Verlag $\mathrm{GmbH}$, ein Teil von Springer Nature - all rights reserved 2020

Eine soeben erschienene S3-Leitlinie für die kardiologische Rehabilitation im deutschsprachigen Raum Europas stützt sich im Wesentlichen auf mehrere durch die DGPR (Deutsche Gesellschaft für Prävention und Rehabilitation von Herz-Kreislauferkrankungen e. V.) und die Sektion Secondary Prevention and Rehabilitation der EAPC (European Association of Preventive (ardiology) initiierte und verfasste Metaanalysen oder Positionspapiere. Demnach besteht für Patienten mit koronarer Herzkrankheit (KHK), Herzinsuffizienz, Klappenvitien und/oder psychologischer Komorbidität eine 1AEmpfehlung, da Mortalität und Rehospitalisierungen gesenkt oder die Lebensqualität sowie depressive Symptome verbessert werden. Für die berufliche Wiedereingliederung liegen jetzt auch standardisierte Handlungsempfehlungen vor.

\section{S3-Leitlinie Kardiologische} Rehabilitation im deutschsprachigen Raum Europas

Erstmals ist eine Leitlinie (LL) zur kardiologischen Rehabilitation (KardReha) auf dem S3-Niveau, also evidenzbasiert, auf der Basis einer strukturierten Literaturrecherche mit metaanalytischer Auswertung in deutscher Sprache publiziert worden [15]. Neben der federführenden DGPR und unter methodischer Betreuung durch die AWMF (Arbeitsgemeinschaft der Wissenschaftlichen Medizinischen Fachgesellschaften e. V.) haben 6 weitere deutsche Fachgesellschaften (DGK [Deutsche Gesellschaft

\author{
Heinz Völler ${ }^{1,2} \cdot$ Bernhard Schwaab ${ }^{3}$ \\ ${ }^{1}$ Klinik am See - Reha-Fachklinik für Innere Medizin, Rüdersdorf bei Berlin, Deutschland \\ ${ }^{2}$ Rehabilitationsmedizin, Universität Potsdam, Potsdam, Deutschland \\ ${ }^{3}$ Curschmann Klinik, Klinikgruppe Dr. Guth GmbH \& Co. KG, Timmendorfer Strand, Deutschland
}

\title{
Kardiologische Rehabilitation
}

für Kardiologie - Herz und Kreislaufforschung e. V.], DGTHG [Deutsche Gesellschaft für Thorax-, Herz- und Gefäßchirurgie], DGSP [Deutsche Gesellschaft für Sportmedizin und Prävention e. V.], DKPM [Deutsches Kollegium für Psychosomatische Medizin e. V.], DGRW [Deutsche Gesellschaft für Rehabilitationswissenschaften e. V.], BNK [Bundesverband Niedergelassener Kardiologen e. V.]) sowie die kardiologischen Gesellschaften aus Österreich und der Schweiz diese LL konsentiert [15]. Über die Deutsche Herzstiftung wurden darüber hinaus Patientenvertreter im Sinne einer partizipativen Entscheidungsfindung eingebunden.

In dieser LL wird die Evidenz der KardReha nicht nur für die Hauptindikationen KHK, Herzinsuffizienz und Vitien beschrieben. Auch seltenere Reha-Indikationen wie EMAH (Erwachsene mit angeborenen Herzfehlern), VAD (,ventricular assist device“), Myokarditis, pAVK (periphere arterielle Verschlusskrankheit), Lungenembolie und pulmonale Hypertonie werden evaluiert. Darüber hinaus wird die Evidenz der rehabilitativen Therapie wie körperliches Training, Ernährung, Entspannung, psychosoziale Intervention für die verschiedenen Indikationen evaluiert. Die Evidenz von Schulungen zur Lebensstilintervention wird aufgezeigt und die Bedeutung der beruflichen Wiedereingliederung hervorgehoben [15].

\section{Koronare Herzerkrankung}

Für keine andere Erkrankung ist die Wirksamkeit einer KardReha so gut belegt wie für die KHK. Dieses trifft auch im Zeitalter akuter Revaskularisations- therapie mit 24-h-PCI („percutaneous coronary interventions") und moderner Pharmakotherapie zu [8]. In einervon der DGPR initiierten Metaanalyse konnte anhand von nach 1995 erschienenen Studien mit einer Beobachtungsdauer von mindestens 6 Monaten an $219.702 \mathrm{~Pa}$ tienten in 25 Studien nachgewiesen werden, dass die Teilnahme an einer KardReha die Gesamtsterblichkeit bei Patienten nach akutem Koronarsyndrom (ACS) um über $60 \%$ (HR [Hazard Ratio] 0,37; 95\%-CI [Konfidenzintervall] 0,20-0,69), nach ACVB(aortokoronarer Venenbypass)-Operation um nahezu $40 \%$ (HR 0,62; $95 \%$-CI $0,54-0,70$ ) sowie in gemischten Populationen bis zu $50 \%$ reduziert [8]. Diese Resultate stimmen prinzipiell mit denen der jüngsten Cochrane-Analyse überein [2]. Hier betrug die Reduktion der kardiovaskulären Mortalität (RR [relatives Risiko] 0,74; 95\%-CI 0,64-0,86) sowie der Gesamtsterblichkeit (OR [Odds Ratio] 0,74; $95 \%$-CI 0,58-0,95) $26 \%$.

\section{》) Die Teilnahme an einer KardReha senkt die Gesamtsterblichkeit bei Patienten nach ACS deutlich}

Die Arbeiten unterscheiden sich jedoch methodisch erheblich. Während die Autoren des Cochrane Reviews nur randomisierte Studien, zum Teil aus den 1970er- und 1980er-Jahren des letzten Jahrhunderts eingeschlossen haben, wurden in der Metaanalyse von Rauch et al. auch kontrollierte Kohortenstudien (prospektiv wie auch retrospektiv) berücksichtigt, deren Ergebnisse auf 
Hier steht eine Anzeige.

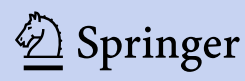


der Propensitiy-based-Matched-Analyse beruhen und damit eher dem Versorgungsalltag entsprechen. Zudem wurden ausschließlich Studien eingeschlossen, die die Kriterien einer multimodalen Rehabilitation mit professioneller Beratung zur Lebensstiländerung einschließlich Nikotinverzicht und Ernährungsberatung beinhalteten, während das Cochrane-Review sich vorwiegend auf Studien zur trainingsbasierten Rehabilitation stützt.

Für die Hochrisikopopulation koronarkranker Diabetiker wurde soeben eine Arbeit zum standardisierten Management bei Patienten mit Typ-2-Diabetes mellitus in der kardiologischen Rehabilitation publiziert, das Teil des von der Europäischen Gesellschaft für Kardiologie entwickelten Diabetes-Programms ist [6].

\section{Patienten mit systolischer Herzinsuffizienz}

Die Therapieziele bei Patienten mit Herzinsuffizienz werden in allen Leitlinien der internationalen Fachgesellschaften gleich formuliert: Verbesserung der körperlichen Belastbarkeit und der Lebensqualität, Vermeidung von Rehospitalisierung sowie Reduktion der Mortalität. Neben der Optimierung der Herzinsuffizienzmedikation sind zur Erreichung dieser Ziele weitere Maßnahmen erforderlich. Auch darin stimmen alle Leitlinien überein [15]. Die Vermittlung der prognostischen Bedeutung einer hohen Adhärenz zur medikamentösen Therapie, ein adäquates körperliches Training, Schulung im Umgang mit der Erkrankung (Gewichtskontrolle, Anpassung der Diuretika), die Unterstützung bei der Krankheitsverarbeitung und der psychischen Stabilisierung (Angst und Depression) haben großen Einfluss auf den Verlauf der Erkrankung und die Lebensqualität der Patienten mit Herzinsuffizienz [15].

Eine aktuelle Metaanalyse aus Deutschland schloss erstmals nur Studien ein, deren Patienten mit einer effektiven Herzinsuffizienzmedikation behandelt worden waren [3]. Von 12.229 Publikationen konnten lediglich 26 RCT (randomisiert kontrollierte Studien) in die Analyse aufgenommen werden. Die
Teilnahme an einer KardReha verbesserte den primären Endpunkt Gesamtmortalität nicht signifikant (HR 0,89; 95\%CI 0,74-1,07). Auch die sekundären Endpunkte Rehospitalisierung (aus allen Gründen und wegen Herzinsuffizienz) sowie die kardiovaskuläre Mortalität wurden nicht signifikant reduziert. Jedoch wurde die körperliche Belastbarkeit, gemessen als maximale Sauerstoffaufnahme durch die Teilnahme an einer KardReha hochsignifikant verbessert sowohl nach 6 Monaten (11 Studien: MD ["mean deviation“] 5,50; 95\%-CI 4,93-6,07) und nach 12 bis 14 Monaten (6 Studien: MD 4,60; $95 \%$-CI 2,22-6,98). Auch die Lebensqualität, gemessen mittels KCCQ (Kansas City Cardiomyopathy Questionnaire), war nach 6 Monaten signifikant besser (2 Studien: MD +1,94; $95 \%$-CI $+0,35-3,56$ ). Dieser Effekt ließ sich allerdings nach 12 Monaten (KCCQ, 1 Studie) nicht mehr statistisch sichern [4].

In einer großen italienischen Beobachtungsstudie nahmen von $140.552 \mathrm{~Pa}$ tienten mit Herzinsuffizienz $39.709 \mathrm{~Pa}-$ tienten (29\%), Alter $75 \pm 11$ Jahre, $47 \%$ Frauen, an einer KardReha teil. In der multivariaten Analyse waren nach 7 Jahren die Gesamtmortalität um über $40 \%$ (HR 0,$5768 ; 95 \%$-CI $0,5650-0,5888$; $p<0,0001)$ und die Rehospitalisierung um $20 \%$ (HR 0,7997; $95 \%$-CI $0,7758-0,8244 ; p<0,0001)$ in der Gruppe mit KardReha im Vergleich zu den Patienten mit "usual care“ signifikant reduziert [14]. Es gab jedoch signifikante und klinisch relevante Unterschiede zwischen beiden Gruppen, und die Zuteilung erfolgte nicht randomisiert. Diese Kohortenstudie repräsentiert den Versorgungsalltag zwar sehr gut. Sie ist dadurch jedoch trotz ihrer großen Patientenzahl wissenschaftlich von eingeschränkter Aussagekraft [14].

\section{》) Bei systolischer Herzin- suffizienz werden körperliche Belastbarkeit und Lebensqualität signifikant verbessert}

Zusammenfassend ist die signifikante Verbesserung der körperlichen Belast- barkeit und der Lebensqualität durch die Teilnahme an einer KardReha bei Patienten mit Herzinsuffizienz wissenschaftlich sehr gut belegt. Die Studienergebnisse zur Verringerung von Morbidität und Mortalität sind jedoch heterogen. Eine KardReha wird bei Patienten mit Herzinsuffizienz dennoch mit hohem Evidenzgrad empfohlen, weil sie besonders geeignet ist, die Titration der prognostisch relevanten Medikamente umzusetzen und ein evidenzbasiertes körperliches Trainingsprogramm zu initiieren [15]. Dies gilt in gleicher Weise für die Patienten nach VAD-Implantation [10].

\section{Rehabilitation nach Herz- klappenoperation und -intervention}

Patienten nach Herzklappenoperationen oder -interventionen stellen ein sehr heterogenes Patientenkollektiv dar. Es kommen junge Patienten mit angeborenen Herzfehlern (EMAH) nach operativer Korrektur ebenso wie über 90-jährige Patienten nach transfemoralem Aortenklappenersatz (TAVI) zur Aufnahme. Wissenschaftliche Studien zur Evidenz der KardReha bei diesen Entitäten lagen bisher jedoch kaum vor [15]. Erst in jüngster Zeit konnten 2 Studien eine signifikante Reduktion von Morbidität und Mortalität bei Patienten nach Herzklappenoperation und nach TAVI durch die Teilnahme an einer KardReha nachweisen [4, 7].

\section{》) KardReha reduziert bei Patienten nach Klappenkorrektur, insbes. nach TAVI, die Mortalität}

In einer Kohortenstudie aus Deutschland wurden 1017 Patienten nach TAVI, $81 \pm 6$ Jahre, $55 \%$ Frauen, LVEF (linksventrikuläre Ejektionsfraktion) $53 \pm 13 \%$, über 6 Monate beobachtet [4]. Obwohl die Teilnahme an einer Rehabilitation allen Patienten angeboten wurde, lehnten $366(36,0 \%)$ eine Teilnahme überwiegend aus persönlichen Gründen ab. Diese Kohorte diente als Kontrollgruppe. Von den 651 Patienten, die an einer Rehabilitation teilnahmen, ab- 
solvierten 435 Patienten $(41,8 \%)$ eine kardiologische und $216(21,2 \%)$ eine geriatrische Rehabilitation [4]. Nach 6 Monaten war das Gesamtüberleben in der Reha-Gruppe signifikant höher als in der Kontrollgruppe (95,0\% vs. $89,8 \% ; p=0,003)$. Auch nach multivariater Analyse blieb der Unterschied mit einer Mortalitätsreduktion von ca. $50 \%$ signifikant (OR 0,49; $95 \%$-CI 0,25-0,94; $p=0,032)$.

Nach getrennter Auswertung war nur die Teilnahme an einer KardReha mit einem verbesserten Überleben nach 6 Monaten assoziiert (OR 0,31; $95 \%$-CI $0,14-0,71 ; p=0,006)$. Die Patienten in der kardiologischen Rehabilitation waren jedoch jünger (80,2 vs. $81,7$ Jahre; $p=0,003)$, häufiger zuvor mit einer aortokoronaren Koronarbypassoperation (ACB-Op.) versorgt (17,8 vs. $9,3 \% ; p=0,004)$, hatten eine niedrigere NYHA(New York Heart Association)Klasse ( 82,0 vs. $88,7 \% ; p=0,049)$ und einen niedrigeren logistischen EuroScore I ( 16,0 vs. $19,0 \% ; p=0,005)$, waren nach der Implantation kürzer hospitalisiert ( 8 vs. 10 Tage; $p<0,001)$ und hatten niedrigere NT-proBNP-Spiegel (1063 vs. $2562 \mathrm{pg} / \mathrm{ml} ; p<0,001)$. Insofern ist der Überlebensvorteil nicht in der Rehabilitationsform, sondern in der Auswahl der Patienten begründet [4].

In einer weiteren Kohortenstudie aus den USA wurden 41.369 Patienten nach offener Herzklappenchirurgie evaluiert (Aortenklappe 68\%, Mitralklappe 22\%, Trikuspidalis $1 \%$, mehrere Klappen 9\%). An einer KardReha nahmen 17.855 Patienten (43,2\%), 73 Jahre (Spanne 68 bis 78 Jahre), $38 \%$ Frauen, teil. Das Angebot einer Rehabilitationsmaßnahme wurde von Patienten mit gleichzeitiger ACBOp. signifikant häufiger wahrgenommen (OR 1,26; 95\%-CI 1,20-1,31). Die mediane Zeit zwischen Entlassung aus der herzchirurgischen Klinik und Beginn des Reha-Programmes betrug 44 Tage (IQR [,interquartile range"] 29 bis 66 Tage) [7].

Die Teilnahme an einer KardReha war mit einer absoluten Reduktion der Mortalität um 4,2\% nach 12 Monaten assoziiert. Auch nach multivariater Korrektur war der Unterschied hochsignifikant (HR 0,39; $95 \%$-CI 0,35-0,44; $p<0,001)$. Die

Kardiologe 2020 · 14:106-112 https://doi.org/10.1007/s12181-020-00384-2

(c) Deutsche Gesellschaft für Kardiologie - Herz- und Kreislaufforschung e.V. Published by Springer Medizin Verlag GmbH, ein Teil von Springer Nature - all rights reserved 2020

\section{H. Völler · B. Schwaab}

\section{Kardiologische Rehabilitation}

\section{Zusammenfassung}

Hintergrund. Eine Verlängerung der Lebensund Arbeitszeit erfordert einen aktiven Lebensstil, eine Optimierung von kardiovaskulären Risikofaktoren und psychosoziale Unterstützung chronisch Herzkranker. Fragestellung. Können die Prognose und Lebensqualität sowie die soziale oder berufliche Teilhabe kardiovaskulär Erkrankter durch kardiologische Rehabilitation (KardReha) verbessert werden?

Material und Methode. Auf der Grundlage neuer Metaanalysen und aktueller Positionspapiere gibt die S3-Leitlinie zur kardiologischen Rehabilitation evidenzbasierte Empfehlungen.

Ergebnisse. Eine KardReha reduziert bei Patienten nach akutem Koronarsyndrom, nach PCI („percutaneous coronary interventions“) oder nach aortokoronarer Koronarbypassoperation (ACB-Op.) sowie nach Klappenkorrektur die Gesamtsterblichkeit. Bei Patienten mit systolischer Herzinsuffizienz (HFrEF [,heart failure with reduced ejection fraction"]) werden Belastbarkeit und Lebensqualität durch eine KardReha verbessert. Psychosozialer Distress kann verringert und die berufliche Wiedereingliederung besser strukturiert werden.

Schlussfolgerung. Im Jahr 2019 liegen aktuelle, evidenzbasierte Leitlinien vor, die aufgrund verbesserter Prognose, Belastbarkeit und Lebensqualität eine multimodale kardiologische Rehabilitation bei Patienten nach akutem kardialem Ereignis auch bei technischem Fortschritt (z. B. katheterbasierter Klappenkorrektur) und unter Aspekten der sozialen und beruflichen Teilhabe empfehlen.

Schlüsselwörter Koronare Herzerkrankung · Herzinsuffizienz . Herzklappenkorrektur . Psychosozialer Distress · Lebensqualität

\section{Cardiac rehabilitation}

Abstract

Background. Prolonging the life span and working life requires an active lifestyle, optimization of cardiovascular risk factors and psychosocial support for patients suffering from chronic heart disease.

Objective. Is it possible to improve the prognosis and quality of life as well as social and occupational participation of patients with cardiovascular diseases by cardiac rehabilitation?

Material and methods. The $\mathrm{S} 3$ guidelines on cardiac rehabilitation in Germanspeaking countries provide evidence-based recommendations based on recent metaanalyses and current position papers.

Results. Cardiac rehabilitation is able to reduce overall mortality in patients with acute coronary syndrome, after percutaneous coronary interventions or surgical revascularization as well as after heart valve correction. In patients with systolic heart failure (heart failure with reduced ejection fraction, HFrEF) exercise capacity and quality of life are improved by cardiac rehabilitation. Psychosocial distress can be reduced and occupational reintegration can be adequately planned.

Conclusion. In 2019 current evidence-based guidelines are available that recommend a multimodal cardiac rehabilitation in patients after an acute cardiac event, due to improvement of prognosis, exercise capacity and quality of life as well as due to technical progress (e.g. catheter-based valve correction) and with respect to social and professional participation.

\section{Keywords}

Coronary artery disease $\cdot$ Heart failure $\cdot$ Heart valve correction. Psychosocial distress . Quality of life 
Rehospitalisierungsrate wurde durch die Teilnahme an der KardReha innerhalb von 12 Monaten in allen Gruppen gleich reduziert: nach Aortenklappenchirurgie HR 0,39; $95 \%$-CI 0,34-0,45; nach Mitralklappenrekonstruktion HR 0,33; 95 \%-CI 0,22-0,51; nach Mitralklappenersatz HR 0,38 ; $95 \%$-CI 0,29-0,50 sowie nach Operation an mehreren Klappen HR 0,44; 95\%-CI 0,34-0,62. Die Reduktion der Mortalität war gleich zwischen den Gruppen mit/ohne begleitende ACB-Op. und unabhängig davon, ob die KardReha sofort nach Operation oder erst später begonnen wurde [7].

Eine Leitlinie, in der die kardiologische Rehabilitation nach Herzklappenoperation oder -intervention wissenschaftlich bewertet wird, liegt jetzt erstmals vor [15]. Neben Reduktion von Mortalität und Morbidität sind Schulungsmaßnahmen zur Endokarditisprophylaxe und zur Antikoagulation (evtl. mit INR[International Normalized Ratio]-Selbstbestimmung) ebenso wie die adäquate berufliche Wiedereingliederung insbesondere der jungen Patienten mit angeborenen Herzfehlern (EMAH) und die Behandlung der zunehmenden Komorbidität der älteren Patienten wichtige Inhalte der KardReha nach Herzklappeneingriffen. Daher wird in der S3-Leitlinie eine kardiologische Rehabilitation nach Vitienkorrektur (Ersatz, Rekonstruktion und Intervention) generell empfohlen [15].

\section{Psychosoziale Intervention in der Rehabilitation}

Wesentliche Bestandteile einer multimodalen KardReha sind psychosoziale Interventionen. In einer ebenfalls von der DGPR initiierten und jüngst veröffentlichten Metaanalyse konnten bei allerdings großer methodischer Vielfalt und eher niedriger bis mäßiger Studienqualität lediglich geringe Effekte nachgewiesen werden [1]. Von 15.373 zwischen Januar 1995 und Oktober 2017 erschienenen Publikationen erfüllten 20 Studien mit definierten Interventionen die vorher festgelegten Ein- und Ausschlusskriterien. Bei 4450 Patienten mit koronarer Herzerkrankung (88,5\%) oder Herzinsuffizienz (11,5\%) konnten im Trend depressive Symptome (MeanStandarddifferenz -0,13; 95\%-CI -0,30, $p=0,05)$ reduziert werden. Die Risikoreduktion für kardiale Ereignisse mit $26 \%$ (RR 0,74; 95\%-CI 0,51-1,07; $p=0,50$ ) über einen Beobachtungszeitraum von 5 Jahren verfehlte knapp die Signifikanz. Zusätzliche psychologische Lebensstilinterventionen oder ein Distress-Management hatten keinen Einfluss auf Ängstlichkeit und Lebensqualität sowie die kardiovaskuläre oder die Gesamtsterblichkeit.

》) Psychosoziale Interventionen während KardReha in der heutigen Form zeigen geringe klinische Effekte

Werden bei Patienten mit koronarer Herzerkrankung (sowohl akutes als auch chronisches Koronarsyndrom) oder Herzinsuffizienz psychologische Interventionen unabhängig von einer Rehabilitation im Vergleich zur Regelversorgung eingesetzt, so kann nach den Ergebnissen eines systematischen Cochrane Reviews an Daten von 35 randomisierten Studien eine signifikante Reduktion der kardiovaskulären Mortalität um $21 \%$ im mittleren Followup über 12 Monate (RR 0,79; 95\%CI $0,63-0,98 ; p=0,04)$ nachgewiesen werden [11]. Darüber hinaus konnten depressive Symptome und Angst ebenfalls verbessert werden.

Ähnlich wie die Verfasser der DGPRMetaanalyse kommen auch die Autoren des Cochrane Reviews zu dem Schluss, dass psychosoziale Interventionen bei Patienten mit kardiovaskulären Erkrankungen einen positiven Effekt auf depressive Symptome und kardiovaskuläre Mortalität haben können. Über die Art und Technik von angewandten Interventionen sowie in welchem Krankheitsstadium bzw. bei welcher Krankheitsausprägung diese angewandt werden sollen, besteht noch Unklarheit.

\section{Berufliche Wiedereingliederung}

Neben der Optimierung der kardiovaskulären Risikofaktoren und der psy- chischen Stabilisierung ist bei Patienten im Erwerbsleben die berufliche Wiedereingliederung vorrangiges Ziel der Rehabilitation. Diese ist besonders erschwert, wenn Teilnehmer einer KardReha zusätzlich eine besondere berufliche Problemlage (BBPL) aufweisen, die bei über $40 \%$ der Teilnehmer vorliegen kann [12]. Nach Daten des kürzlich erschienenen europaweiten Registers (EuroAspire IV: European Action on Secondary and Primary Prevention through Intervention to reduce Events) an 3291 berufstätigen Patienten liegt die Rate der beruflichen Wiedereingliederung nach 13,3 Monaten bei $76 \%$, bei Patienten mit BBPL jedoch unter $50 \%$ [5, 13]. Um die Rate der beruflichen Wiedereingliederung von Patienten mit BBPL zu erhöhen, wurde in einer randomisierten Untersuchung die Effektivität einer intensiven sozialarbeiterischen Intervention während 3-wöchiger stationärer Rehabilitation untersucht, die im Hinblick auf die Rate beruflicher Wiedereingliederung nach 12 Monaten einen neutralen Effekt aufwies (OR 0,94; $95 \%$-CI $0,52-1,609 ; p=0,820)$. Eine vor der Erkrankung bestehende Arbeitslosigkeit (OR 0,13; 95\%-CI 0,06-0,33; $p<0,001)$ sowie erhöhte Werte einer bestehenden Ängstlichkeit zum Zeitpunkt der Entlassung aus der Rehabilitation (OR 0,15; 95\%-CI 0,06-0,37; $p<0,001$ ) waren demgegenüber die bestimmenden Faktoren einer nicht erfolgreichen beruflichen Wiedereingliederung [5].

Um kardiovaskuläre Patienten hinsichtlich ihrer Arbeitsfähigkeit besser charakterisieren und sie in einem standardisierten Behandlungspfad bedarfsgerecht durch die einzelnen Berufsgruppen (Sozialarbeiter, Haus- oder Betriebsarzt, Kardiologen, Psychologen) behandeln zu können, wurde von der Sektion Secondary Prevention and Rehabilitation der European Association of Preventive Cardiology (EAPC) eine Übersichtsarbeit verfasst [9]. Diese sollte auch $\mathrm{zu}$ einer internationalen Harmonisierung der Abläufe in Rehabilitationseinrichtungen beitragen und in Zukunft multizentrische Untersuchungen ermöglichen. 
Hier steht eine Anzeige.

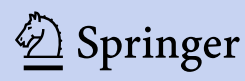




\section{Fazit für die Praxis}

- Die Teilnahme an einer kardiologischen Rehabilitation (KardReha) reduziert bei Patienten mit koronarer Herzkrankheit (KHK) die Gesamtsterblichkeit.

- Patienten mit Herzinsuffizienz weisen nach KardReha eine (signifikant) verbesserte Belastbarkeit und Lebensqualität auf.

- Neben einer Senkung der Mortalität kann durch KardReha bei Patienten nach Klappenkorrektur der nutritive und funktionelle Status verbessert werden.

- Psychische Komorbiditäten wie Depression und Angst können durch KardReha verbessert werden.

- Zur Standardisierung und Verbesserung der beruflichen Wiedereingliederung nach kardiovaskulärem Indexereignis wurden Handlungsempfehlungen verfasst.

\section{Korrespondenzadresse}

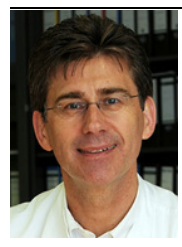

Prof. Dr. med. Heinz Völler Klinik am See - RehaFachklinik für Innere Medizin Seebad 84, 15562 Rüdersdorf bei Berlin, Deutschland heinz.voeller@ klinikamsee.com

Prof. Dr. med.
Bernhard Schwaab
Curschmann Klinik,
Klinikgruppe Dr. Guth GmbH
\&Co. KG
Saunaring 6, 23669 Timmen-
dorfer Strand, Deutschland
prof.schwaab@drguth.de

\section{Einhaltung ethischer Richtlinien}

Interessenkonflikt. H. Völler und B. Schwaab geben an, dass kein Interessenkonflikt besteht.

Für diesen Beitrag wurden von den Autoren keine Studien an Menschen oder Tieren durchgeführt Für die aufgeführten Studien gelten die jeweils dort angegebenen ethischen Richtlinien.

\section{Literatur}

1. Albus $C$, Herrmann-Lingen $C$, Jensen $K$, Hackbusch $M$, Münch N, Kuncewicz $C$, Grilli $M$
Schwaab B, Rauch B, German Society of Cardiovascular Prevention \& Rehabilitation (DGPR) (2019) Additional effects of psychological interventions on subjective and objective outcomes compared with exercise-based cardiac rehabilitation alone in patients with cardiovascular disease: A systematic review and meta-analysis. Eur J Prev Cardiol 26(10):1035-1049

2. Anderson L, Oldridge N, Thompson DR, Zwisler AD Rees K, Martin N, Taylor RS (2016) Exercise-based cardiac rehabilitation for coronary heart disease: Cochrane Systematic Review and meta-analysis. J Am Coll Cardiol 67(1):1-12

3. Bjarnason-Wehrens B, Nebel R, Jensen Ketal (2019) Exercise-based cardiac rehabilitation in patients with reduced left ventricular ejection fraction: the Cardiac Rehabilitation Outcome Study in Heart Failure (CROS-HF): A systematic review and metaanalysis. Eur J Prev Cardiol. https://doi.org/10. $1177 / 2047487319854140$

4. Butter C, Groß J, Haase-Fielitz A et al (2018) Impact of rehabilitation on outcomes after TAVI: a preliminary study. J Clin Med. https://doi.org/10. 3390/jcm7100326

5. Cauter JV, Bacquer D, Clays E, Smedt D, Kotseva K Braeckman L (2019) Return to work and associations with psychosocial well-being and healthrelated quality of life in coronary heart disease patients: results from EUROASPIRE IV. Eur J Prev Cardiol 26(13):1386-1395

6. Hansen D, Kraenkel N, Kemps $H$, Wilhelm M, Abreu A, Pfeiffer AF, Jordão A, Cornelissen V, Völler H (2019) Management of patients with type 2 diabetes in cardiovascular rehabilitation. Eur J Prev Cardiol 26(2_suppl):133-144

7. Patel D, Duncan M, Shah A et al (2019) Association of cardiac rehabilitation with decreased hospitalization and mortality risk after cardiac valve surgery. JAMA Cardiol. https://doi.org/10.1001/ jamacardio.2019.4032

8. Rauch B, Davos CH, Doherty P, Saure D, Metzendorf MI, Salzwedel A, Völler H, Jensen K, Schmid JP 'Cardiac Rehabilitation Section', European Association of Preventive Cardiology (EAPC), Institute of Medical Biometry and Informatics (IMBI), Department of Medical Biometry, University of Heidelberg, Cochrane Metabolic and Endocrine Disorders Group, Institute of General Practice, Heinrich-Heine University (2016) The prognostic effect of cardiac rehabilitation in the era of acute revascularisation and statin therapy: a systematic review and meta-analysis of randomized and non-randomized studies - the cardiac rehabilitation outcome study (CROS). Eur J Prev Cardiol 23(18):1914-1939

9. Reibis R, Salzwedel A, Abreu A, Corra U, Davos C Doehner W, Doherty $P$, Frederix I, Hansen $D_{1}$ Iliou CM, Vigorito C, Völler H, Secondary Prevention and Rehabilitation of the European Association of Preventive Cardiology (EAPC) (2019) The importance of return to work: How to achieve optimal reintegration in ACS patients. Eur J Prev Cardiol 26(13):1358-1369

10. Reiss N, Schmidt T, Langheim E, BjarnasonWehrens B, Marx R, Sindermann JR, Knoglinger E (2019) Inpatient Cardiac Rehabilitation of LVAD Patients-Updated Recommendations from the Working Group of the German Society for Prevention and Rehabilitation of Cardiovascular Diseases. Thorac Cardiovasc Surg. https://doi.org/ 10.1055/s-0039-1691837

11. Richards $\mathrm{SH}$, Anderson $\mathrm{L}$, Jenkinson $\mathrm{CE}$, Whalley $B$ Rees K, Davies P, Bennett P, Liu Z, West R, Thompson DR, Taylor RS (2018) Psychological interventions for coronary heart disease: Cochrane systematic review and meta-analysis. Eur J Prev Cardiol 25(3):247-259

12. Salzwedel A, Reibis R, Hadzic M, Buhlert $H$, Völler H (2019) Patients' expectations of returning to work, co-morbid disorders and work capacity at discharge from cardiac rehabilitation. Vasc Health RiskManag 15:301-308

13. Salzwedel A, Wegscheider K, Schulz-Behrendt C, Dörr G, Reibis R, Völler H (2019) No impact of an extensive social intervention program on return to work and quality of life after acute cardiac event: a cluster-randomized trial in patients with negative occupational prognosis. Int Arch Occup Environ Health 92(8):1109-1120

14. Scalvini S, Grossetti F, Paganoni A et al (2019) Impact of in-hospital cardiac rehabilitation on mortality and readmissions in heart failure: $a$ population study in Lombardy, Italy, from 2005 to 2012. Eur JPrev Cardiol 26:808-817

15. S3-Leitlinie Kardiologische Rehabilitation im deutschsprachigen Raum Europas (D-A-CH). AWMF-Registernummer:133-001.www.awmf.org 